\title{
On twinning and anisotropy in rolled Mg alloy AZ31 under uniaxial tension
}

\author{
Xiaoqian Guo ${ }^{1}$, Adrien Chapuis ${ }^{2}$, Xianbiao Mao ${ }^{1}$, Qing Liu ${ }^{2}$ and Peidong $\mathrm{Wu}^{3, a}$ \\ ${ }^{1}$ State Key Laboratory for Geomechanics and Deep Underground Engineering, China University of Mining and Technology, Xuzhou, \\ Jiangsu 221116, China \\ ${ }^{2}$ College of Materials Science and Engineering, Chongqing University, Chongqing 400044, China \\ ${ }^{3}$ Department of Mechanical Engineering, McMaster University, Hamilton, Ontario, Canada
}

\begin{abstract}
The Elastic Visco-Plastic Self-Consistent (EVPSC) model, with the recently developed Twinning and DeTwinning (TDT) description, is applied to study the mechanical behaviour of hot-rolled Mg alloy AZ31 under uniaxial tension. Numerical results are compared to the experimental uniaxial tensile tests reported earlier by Chapuis et al. [29] for the out-of-plane directions of a thick plate along angles of $\alpha=0^{\circ}, 30^{\circ}, 45^{\circ}, 60^{\circ}$ and $90^{\circ}$ between the normal direction and longitudinal specimen axis. It is shown that accounting for the initial texture and calibrating the EVPSC-TDT model by using uniaxial tension tests along the rolling direction and normal direction permits prediction of the strength anisotropy and strain hardening behavior along all five tensile directions, i.e. for cases in which the contribution of twinning is dominating, negligible or intermediate.
\end{abstract}

\section{Introduction}

Modeling mechanical behaviour of textured magnesium $(\mathrm{Mg})$ alloys has been a hot research topic (see e.g. [123]).However, it is noted that most of the research has been restricted to uniaxial straining along directions of orthotropic symmetry, where the straining is either essentially twinning dominated or essentially devoid of twinning. The works of Oppedal et al. [24] and Guo et al. [25] are exceptional. Oppedal et al. [24] performed uniaxial compression testing along off-axis directions within an extruded AM30 bar. They employed these data to illustrate the need for a new model, which specifically accounts for the dislocation density evolution, as well as the "transmutation" of that dislocation density due to twinning. Guo et al. [25] studied, both experimentally and numerically, the anisotropy in a hot-rolled AZ31 plate under uniaxial compression. The experimental tests covered a wide range of tensile twinning activity: from twinning dominated, through intermediate twinning activity to negligible twinning. The numerical simulations were based on the Elastic Visco-Plastic SelfConsistent (EVPSC) model of Wang et al. [26], with the Twinning and De-Twinning (TDT) description later introduced by Wang et al. [27,28]. It has been demonstrated that accounting for the initial texture and calibrating the EVPSC-TDT model using in-plane uniaxial tension and compression along the rolling direction (RD) permits prediction of the strength anisotropy and monotonic strain hardening behavior along any tilt angle with respect to the normal direction
(ND). It is very interesting to note that this capability is achieved without requiring a complex, dislocation density-based latent hardening approach.

In the present paper, the mechanical anisotropy of hotrolled Mg alloy AZ31 under uniaxial tension is investigated numerically, based on the EVPSC-TDT model. Numerical results are compared with the experimental results reported by Chapuis et al. [29]. In the experimental work, five uniaxial tension tests along angles of $\alpha=0^{\circ}, 30^{\circ}, 45^{\circ}, 60^{\circ}$ and $90^{\circ}$ between the ND and longitudinal specimen axis were performed to experimentally study the mechanical anisotropy. The paper is organized as follows. Section 2 introduces the EVPSC-TDT model. In Section 3 we show both the experimental and predicted results with emphasizing on relationships between twinning and anisotropy in the sheet under uniaxial tension. Conclusions are drawn in Section 4.

\section{Constitutive Model}

In this section, we briefly recapitulate the EVPSC-TDT model. For details, we refer to Wang et al. [26,28]. The plastic deformation of a crystal is assumed to be due to crystallographic slip and twinning on crystallographic system $\left(\boldsymbol{s}^{\alpha}, \boldsymbol{n}^{\alpha}\right)$, with $\boldsymbol{s}^{\alpha}$ and $\boldsymbol{n}^{\alpha}$ being the slip/twinning direction and the normal of the slip/twinning plane for system $\alpha$, respectively. For $\mathrm{Mg}$ alloys, Basal $\langle a\rangle$, Prismatic $\langle a\rangle$, and Pyramidal $<c+a>$ slip systems, and $\{10 \overline{1} 2\}<\overline{1} 011>$ extension twin system are usually considered.

\footnotetext{
${ }^{\mathrm{a}}$ Corresponding author: peidong@mcmaster.ca
} 
Regardless of slip or twinning, the driving force for shear rate, $\dot{\gamma}^{\alpha}$, is the resolved shear stress, $\tau^{\alpha}$. For slip,

$$
\dot{\gamma}^{\alpha}=\dot{\gamma}_{0}\left|\tau^{\alpha} / \tau_{c r}^{\alpha}\right|^{\frac{1}{m}} \operatorname{sgn}\left(\tau^{\alpha}\right)
$$

For twinning,

$$
\dot{\gamma}^{\alpha}=\left\{\begin{array}{cc}
\dot{\gamma}_{0}\left|\tau^{\alpha} / \tau_{c r}^{\alpha}\right|^{\frac{1}{m}} & \tau^{\alpha}>0 \\
0 & \tau^{\alpha} \leq 0
\end{array}\right.
$$

where $\dot{\gamma}_{0}$ is a reference shear rate, $\tau_{c r}^{\alpha}$ is the critical resolved shear stress (CRSS), and $m$ is the strain rate sensitivity. The TDT model assumes that a grain has four potential operations associated with twinning and detwinning. Operation $\mathrm{A}$ is twin nucleation and initiates a twin band or 'child'. Operation B is a propagation of the child into the parent grain. Operations A and B increase the twin volume fraction and thus correspond to twinning. Operation $\mathrm{C}$ is a propagation of the parent into the child. Operation D splits the twin band and decreases the twin volume fraction through re-twinning. Operations $\mathrm{C}$ and $\mathrm{D}$ decrease the twin volume fraction and thus correspond to detwinning. Furthermore, the TDT model treats new twin band (child) as a new grain, and all twin variants are possibly present.

For both slip and twinning, the evolution of CRSS, $\tau_{c r}^{\alpha}$, is given by:

$$
\dot{\tau}_{c r}^{\alpha}=\frac{d \hat{\tau}^{\alpha}}{d \Gamma} \sum_{\chi} h^{\alpha \chi}\left|\dot{\gamma}^{\chi}\right|
$$

where $\Gamma=\sum_{\beta} \int\left|\dot{\gamma}^{\alpha}\right| d t$ is the accumulated shear strain in the grain, and $h^{\alpha \chi}$ are the latent hardening coupling coefficients, which empirically account for the obstacles on system $\alpha$ associated with system $\chi \cdot \hat{\tau}^{\alpha}$ is the threshold stress and is characterized by:

$$
\hat{\tau}^{\alpha}=\tau_{0}^{\alpha}+\left(\tau_{1}^{\alpha}+h_{1}^{\alpha} \Gamma\right)\left(1-\exp \left(-\frac{h_{0}^{\alpha}}{\tau_{1}^{\alpha}} \Gamma\right)\right)
$$

Here, $\tau_{0}, h_{0}, h_{1}$, and $\tau_{0}+\tau_{1}$ are the initial CRSS, the initial hardening rate, the asymptotic hardening rate, and the back-extrapolated CRSS, respectively.

Because it is rare that a grain can be fully twinned, a threshold twin volume fraction is defined in the model to terminate twinning. Consequently, the TDT model introduces two statistical variables: accumulated twin fraction, $V^{a c c}$, and effective twinned fraction, $V^{\text {eff }}$. More specifically, $V^{a c c}$ and $V^{e f f}$ are the weighted volume fraction of the twinned region and volume fraction of twin terminated grains, respectively. The threshold volume fraction, $V^{\text {th }}$, is defined as
$V^{\text {th }}=\min \left(1.0, A_{1}+A_{2} \frac{V^{e f f}}{V^{a c c}}\right)$, where $A_{1}$ and $A_{2}$ are two material constants.

The response of a polycrystal comprised of many grains is obtained by applying the self-consistent approach. Various self-consistent schemes have been proposed. Wang et al. [26] have demonstrated that the predicted response of a polycrystal is very sensitive to the self-consistent scheme employed. Wang et al. [30] have evaluated various self-consistent schemes used in selfconsistent modeling by applying them to the large strain behavior of magnesium alloy AZ31B plate under different deformation processes. It was found that the Affine self-consistent scheme gave the best overall performance among the self-consistent approaches examined. Therefore, the Affine self-consistent scheme is employed in the present study.

\section{Results and discussion}

The material reported by Chapuis et al.[29] and considered in the present paper is a $50 \mathrm{~mm}$ thick rolled magnesium alloy AZ31, annealed for homogenization at $400^{\circ} \mathrm{C}$ for $1 \mathrm{~h}$. The rolled and annealed plate has an average grain size of about $40 \mu \mathrm{m}$. In the present paper the $\mathrm{RD}$, TD and ND stand for the rolling, transverse and normal directions, respectively. The initial texture of the material was measured using an EBSD map with a step size of $2 \mu \mathrm{m}$. It is shown in Fig. 1 in terms of the $\{0001\}$, $\{11-20\}$ and $\{10-10\}$ pole figures. Clearly, the material exhibits a strong basal texture.
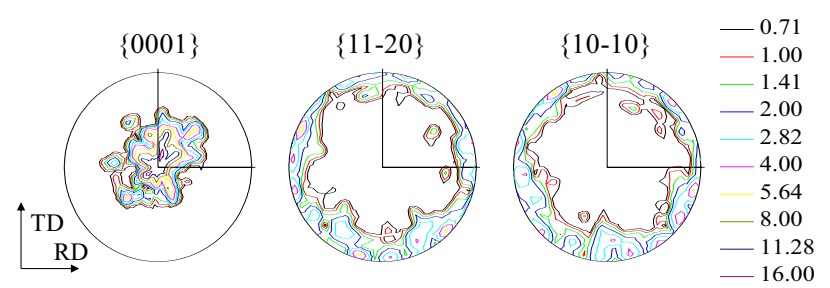

Figure 1. Initial texture presented in terms of the $\{0001\},\{11-$ $20\}$ and $\{10-10\}$ pole figures.

Uniaxial tension tests along different directions with respect to the ND have been performed by Chapuis et al. [29]. More specifically, as shown in Fig. 2, five different specimen orientations with tilt angles of $\alpha=0^{\circ}, 30^{\circ}, 45^{\circ}$, $60^{\circ}$ and $90^{\circ}$ between the ND and loading direction (LD) were used to study the mechanical anisotropy under uniaxial tension. These tests are very similar to those carried out by Wang and Choo [31] on samples with different mean grain sizes in the range of $26 \sim 127 \mu \mathrm{m}$.

In all the simulations reported in the present paper, the reference slip/twinning rate $\dot{\gamma}_{0}$ and the rate sensitivity $m$ are prescribed to be the same for all slip/twinning systems: $\dot{\gamma}_{0}=0.001 s^{-1}$ and $m=0.05$, respectively. The room temperature elastic constants of the magnesium single crystal are taken as $C_{11}=58.0, C_{12}=25.0$, $C_{13}=20.8, C_{33}=61.2$ and $C_{44}=16.6$ (units of $\mathrm{GPa}$ ). Values of the hardening parameters are estimated by 
curve-fitting numerical simulations of monotonic uniaxial tension along the ND and uniaxial tension along the RD to the corresponding experimental data.

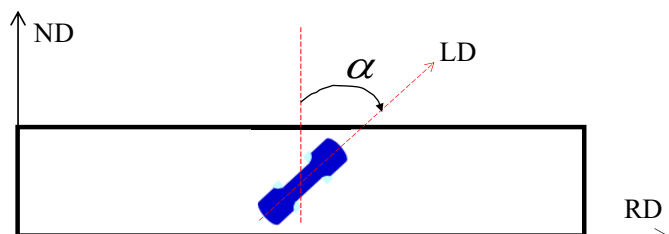

Figure 2. Schematic representation of a uniaxial tension sample with an angle $\alpha$ rotating around the TD from the ND to the loading direction (LD).

Fig. 3 presents the measured and simulated true stress and true strain curves under uniaxial tension along $\alpha=0$ (ND) and $\alpha=90^{\circ}$ (RD). The importance of extension twinning under uniaxial tension along the $\mathrm{ND}\left(\alpha=0^{\circ}\right)$ is clearly revealed by the characteristic $S$-shape of the flow curve, while uniaxial tension along the $\mathrm{RD}\left(\alpha=90^{\circ}\right)$ exhibits a typical slip dominated plastic deformation. It is found that the EVPSC-TDT model can fit the experimental curves very well. The determined values of the material constants are listed in Table 1.

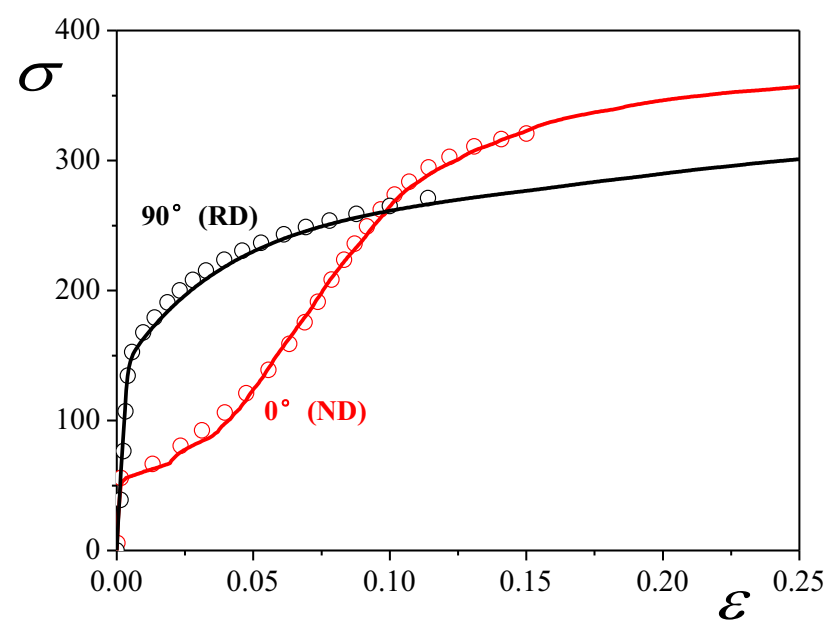

Figure 3. Measured (symbols) and simulated (solid lines) true stress and true strain curves under uniaxial tension along $\alpha=0$ (ND) and $\alpha=90^{\circ}(\mathrm{RD})$. The experimental data are taken from Chapuis et al. [29].

Fig. 4 presents the relative activities of slip/twinning under uniaxial tension along $\alpha=0^{\circ}$ and $\alpha=90^{\circ}$. The relative activities are defined as the ratios of the plastic shear rates of each deformation mode to the total plastic shear rate due to all the deformation modes at the applied strain. Under uniaxial tension along $\alpha=0^{\circ}$ and at strains $\varepsilon \leq 0.04$, tensile twinning is very active, and the remaining plasticity is accommodated by Basal slip. For strains $\varepsilon \geq 0.05$, the tensile twining activity is significantly reduced, and the EVPSC-TDT model predicts increasing Prismatic slip activity and little Pyramidal slip (Fig. 4a). As expected, there is negligible twinning activity under uniaxial tension along $\alpha=90^{\circ}$. The model predicts that, under uniaxial tension along $\alpha=90^{\circ}$, mostly Basal and Prismatic slip accommodate the plastic deformation.
Table 1. List of values of the hardening parameters involved in the EVPSC-TDT model.

\begin{tabular}{|c|c|c|c|c|c|c|c|}
\hline Mode & $\begin{array}{c}\tau_{0} \\
/ \mathrm{MPa}\end{array}$ & $\begin{array}{c}\tau_{1} \\
/ \mathrm{MPa}\end{array}$ & $\begin{array}{c}h_{0} \\
/ \mathrm{MPa}\end{array}$ & $\begin{array}{c}h_{1} \\
/ \mathrm{MPa}\end{array}$ & $h^{s t}$ & $A_{1}$ & $A_{2}$ \\
\hline Basal & 12 & 1 & 10 & 10 & 1 & & \\
\hline Prismatic & 78 & 55 & 650 & 15 & 1.4 & & \\
\hline Pyramidal & 100 & 140 & 2000 & 40 & 1 & & \\
\hline $\begin{array}{c}\text { Extension } \\
\text { twin }\end{array}$ & 35 & 10 & 200 & 10 & 1 & 0.2 & 0.8 \\
\hline
\end{tabular}

(a)
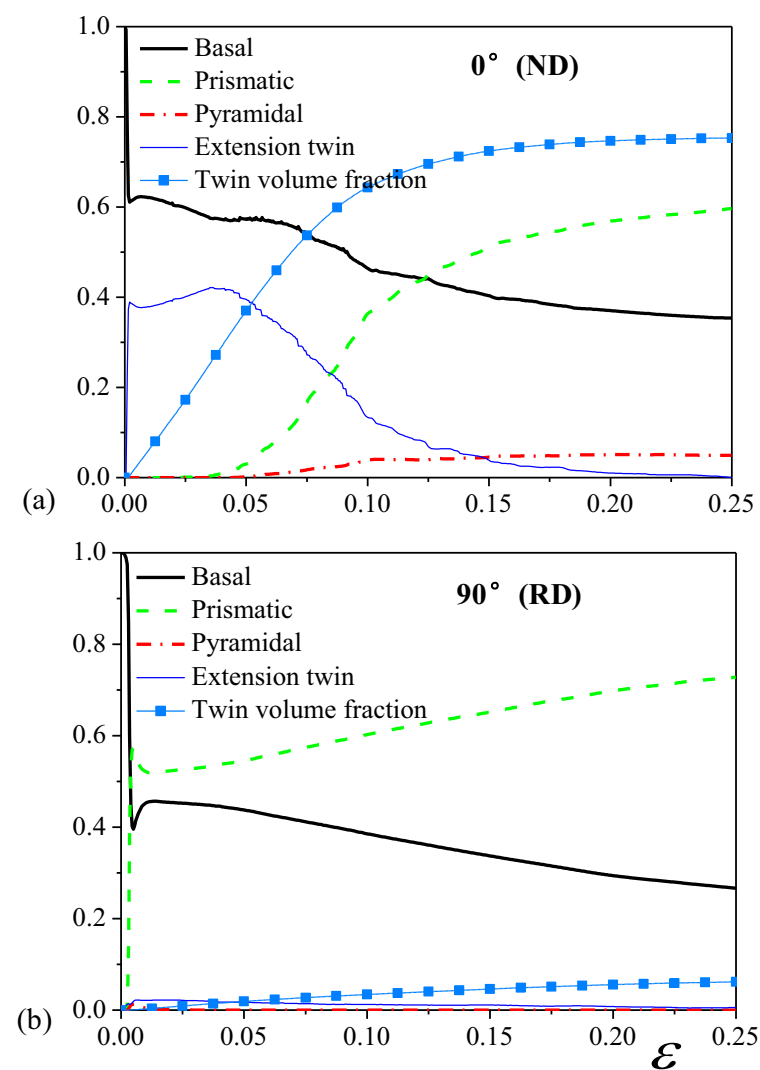

Figure 4. Predicted relative slip and twinning activities and twin volume fraction under uniaxial tension along $\alpha=0^{\circ}$ (a), and $\alpha=90^{\circ}$ (b).

It is noted that Pyramidal slip is almost inactive in uniaxial tension along $\alpha=90^{\circ}$ and little $(<6 \%)$ in uniaxial tension along $\alpha=0^{\circ}$. It is found that Pyramidal slip activity is always below $6 \%$ in all the uniaxial tension tests. This implies that the hardening parameters associated with the Pyramidal slip cannot be confidently determined from the uniaxial tension cases performed in the present study. However, it is also noted that during in-plane uniaxial compression of a rolled plate with strong basal texture, Pyramidal slip is very active at relatively large strains $[4$, 19]. Thus, uniaxial compression allows us to confidently fit the material parameters associated with the Pyramidal slip system [25]. However, in-plane uniaxial compression was not carried out in the work of Chapuis et al.[29]. Nevertheless, based on the previous simulations performed on similar rolled AZ31 plates [19, 25], the values of the material parameters associated with the 
Pyramidal slip system reported in Table 1 are found to be in a reasonably realistic range.
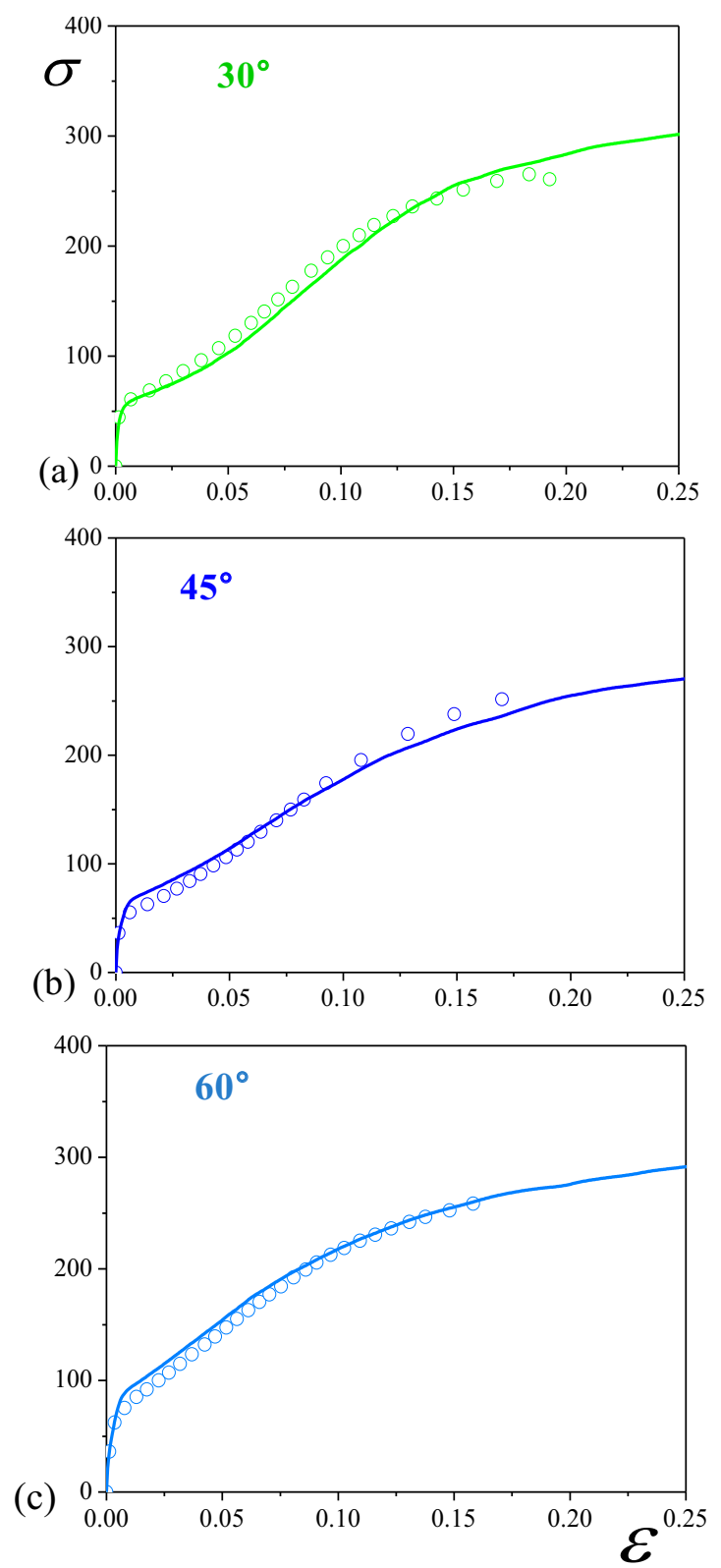

Figure 5. Measured (symbols) and simulated (solid lines) true stress and true strain curves under uniaxial tension along $\alpha=30^{\circ}$ (a), $\alpha=45^{\circ}$ (b), and $\alpha=60^{\circ}$ (c). The experimental data are taken from Chapuis et al. [29].

We proceed by studying uniaxial tension along $\alpha=30^{\circ}$, $\alpha=45^{\circ}$ and $\alpha=60^{\circ}$. Fig. 5 shows the measured and predicted stress and strain curves. In the case of $\alpha=30^{\circ}$ (Fig. 5a), both the measured and predicted stress and strain curves are similar to the case of $\alpha=0^{\circ}$ (i.e. along the ND). An $S$-shaped stress and strain curve indicates that tensile twinning is an important plastic deformation mechanism. The EVPSC-TDT model reproduces very well the measured flow stress. For $\alpha=60^{\circ}$ (Fig. 5c), a "concave-down" stress and strain curve is observed both experimentally and numerically. This implies that the deformation under uniaxial tension along $\alpha=60^{\circ}$ is slip dominated. The agreement between the measured and predicted stress and strain curves is again very good. The uniaxial tension behavior of the $\alpha=45^{\circ}$ case (Fig. 5b) is between those of the $\alpha=30^{\circ}$ and $\alpha=60^{\circ}$ cases. The EVPSCTDT model slightly over-estimates the flow stress at small strains and slightly under-estimates the flow stress at large strain levels.

Although not shown in the present paper, it was found that the EVPSC-TDT model predicts very well the measured texture evolution reported in Chapuis et al. [29].

\section{Conclusions}

We have numerically studied the twinning and anisotropy in a hot-rolled AZ31 plate under uniaxial tension. It has been demonstrated that accounting for the initial texture and calibrating the EVPSC-TDT model by using uniaxial tension along the RD and ND permits prediction of the strength anisotropy and strain hardening behavior along other directions, for cases for which the contribution of twinning is large, small and intermediate. Notably, it is not required to parameterize any complex dislocation density-based hardening model.

\section{Acknowledgements}

X.Q. Guo and X.B. Mao acknowledge the support of the State Key Development Program for Basic Research of China (Grant No. 2013CB227900), the Joint Funds of the National Natural Science Foundation of China (Grant No.U1261201). A. Chapuis is co-supported by the National Basic Research Program of China ("973" Project) (Grantno.2013CB632204) and the National Natural Science Foundation of China (51101175, 51371203 and 51350110332). P.D. Wu is supported by the Natural Sciences and Engineering Research Council of Canada (NSERC).

\section{References}

1. H. Abdolvand, M.R. Daymond. Acta Mater. 60, 2240(2012).

2. S. Graff, W. Brocks, D. Steglich. Int. J. Plasticity 23, 1957(2007).

3. C.F. Gu, L.S. Toth, M. Hoffman. Acta Mater. 62, 212(2014).

4. X.Q. Guo, W. Wu, P.D. Wu, H. Qiao, K. An, P.K. Liaw. Scripta Mater. 69, 319(2013).

5. T. Hama, Y. Kuchinomachi, N. Hosokawa, H. Fujimoto, H. Takuda. Mater. Sci. Eng. A551, 209(2012).

6. J. Jain, P. Cizek, W.J. Poole, M.R. Barnett. Acta Mater. 61, 4091(2013).

7. F. Kabirian, A.S. Khan, T. Gnäupel-Herlod. Int. J. Plasticity 68, 1(2015).

8. J.H. Kim, D. Kim, Y.-S. Lee, M.-G. Lee, K. Chung, H.-Y. Kim, R.H. Wagoner. Int. J. Plasticity 50, 66(2013). 
9. M. Knezevic, A. Levinson, R. Harris, R.K. Mishra, R.D. Doherty, S.R. Kalidindi. Acta Mater. 58, 6230(2010).

10. S.Y. Lee, H. Wang, M.A. Gharghouri, G. Nayyeri, W. Woo, E. Shin, P.D. Wu, W.J. Poole, W. Wu, K. An. Acta Mater.73, 139(2014).

11. J. Levesque, K. Inal, K.W. Neale, R.K. Mishra. Int. J. Plasticity 26, 65(2010).

12. O. Muránsky, M.R. Barnett, D.G. Carr, S.C. Vogel, E.C. Oliver. Acta Mater. 58, 1503(2010).

13. H. Qiao, S.R. Agnew, P.D. Wu. Int. J. Plasticity 65, 61(2015).

14. A. Staroselsky, L. Anand. Int. J. Plasticity 19, 1843(2003).

15. H. Wang, P.D. Wu, M.A. Gharghouri. Mater. Sci. Eng. A527, 3588 (2010).

16. H. Wang, P.D. Wu, K.P. Boyle, K.W. Neale. Int. J. Solids Structures 48, 1000(2011).

17. H. Wang, P.D. Wu, C.N. Tomé, J. Wang. Int. J. Solids Structures 49, 2155(2012).

18. H. Wang, P.D. Wu, J. Wang. Int. J. Plasticity 47, 49(2013).

19. W. Wu, H. Qiao, K. An, X.Q. Guo, P.D. Wu, P.K. Liaw. Int. J. Plasticity 62, 105(2014).

20. P.D. Wu, X.Q. Guo, H. Qiao, D.J. Lloyd. Mater. Sci. Eng. A625, 140(2015).

21. G.W. Zhou, M.K. Jain, P.D. Wu, Y.C. Shao, D.Y. Li, Y.H. Peng. Int. J. Plasticity 79, 19(2016).

22. J.J. Bhattacharyya, F. Wang, P.D. Wu, W. Whittington, H. El Kadiri, S.R. Agnew. Int. J. Plasticity 81, 123(2016).

23. H. Wang, S.Y. Lee, M.A. Gharghouri, P.D. Wu, S.G. Yoon. Acta Mater. 107, 414(2016).

24. A.L. Oppedal, H.El Kadiri, C.N. Tomé, S.C. Vogel, M. Horstemeyer.Philos. Mag. 93, 4311(2013).

25. X.Q. Guo, A. Chapuis, P.D. Wu, S.R. Agnew. Int. J.
Solids Structures 64-65, 42(2015).

26. H. Wang, P.D. Wu, C.N. Tomé, Y. Huang. J. Mech. Phys. Solids 58, 594(2010).

27. H. Wang, P.D. Wu, C.N. Tomé, J. Wang. Mater. Sci. Eng. A555, 93(2012).

28. H. Wang, P.D. Wu, J. Wang, C.N. Tomé. Int. J. Plasticity 49, 36(2013).

29. A. Chapuis, P. Lei, Q. Liu. Mater. Sci. Eng. A561, 167(2013).

30. H. Wang, B. Raeisinia, P.D. Wu, S.R. Agnew, C.N. Tomé. Int. J. Solids Structures 47, 2905(2010).

31. Y. Wang, H. Choo. Acta Mater. 81, 83(2014). 